\title{
Relação entre o escore de comportamento materno e as características fisiológicas de ovelhas ${ }^{1}$
}

\author{
Marta Farias Aita ${ }^{2}$, Vivian Fischer ${ }^{3}$, César Henrique Espírito Candal Poli ${ }^{3}$, Maria Teresa \\ Moreira Osório ${ }^{4}$, Isabella Dias Barbosa Silveira ${ }^{4}$, Marcelo Brasil Selbot ${ }^{5}$, Daniel Bulgareli \\ Montano ${ }^{4}$, Paula Baierle Losekann ${ }^{5}$
}

\footnotetext{
${ }^{1}$ Pesquisa parcialmente financiada pelo CNPq.

2 Programa de Pós-Graduação em Zootecnia da UFRGS.

${ }^{3}$ Departamento de Zootecnia da UFRGS.

${ }^{4}$ Departamento de Zootecnia da UFPel.

${ }^{5}$ Faculdade de Agronomia da UFRGS.
}

RESUMO - Neste estudo objetivou-se avaliar a relação do escore de comportamento materno de ovelhas com os parâmetros fisiológicos relacionados ao seu temperamento. Durante a parição, foram avaliadas 258 ovelhas Corriedale e 50 ovelhas Ideal criadas em campo natural em três fazendas na região sudoeste do estado do Rio Grande do Sul, Brasil. Foram analisados os efeitos propriedade, idade, tipo de parto, escore de comportamento materno e escore de condição corporal antes do parto e ao desmame em relação às frequências cardíaca e respiratória e temperatura corporal dos animais ao desmame. Nas primeiras 24 horas de vida do cordeiro, o escore de comportamento materno foi atribuído à distância de fuga da ovelha em relação ao seu cordeiro: 1) $\geq 10 \mathrm{~m}$ e não retorna ao cordeiro; 2) $\geq 10 \mathrm{~m}$ do cordeiro e retorna; 3) 5 a $10 \mathrm{~m}$; 4) entre $1 \mathrm{e}<5 \mathrm{~m}$; 5) $<1 \mathrm{~m}$; 6) mantém contato físico. Ovelhas com escore de comportamento materno <3 apresentaram maior frequência cardíaca, mas valores semelhantes de frequência respiratória e temperatura corporal se comparadas àquelas com escore de comportamento materno $\geq 3$. Ovelhas criadas em Bagé apresentaram os maiores valores de frequência cardíaca e respiratória. Ovelhas com escore de condição corporal antes do parto $<2,0$ apresentaram menores valores de frequência cardíaca e respiratória e temperatura corporal, enquanto ovelhas com escore de condição corporal ao desmame $<2,0$ tiveram maiores valores de frequência cardíaca e respiratória e menor temperatura corporal. A pouca associação entre os parâmetros fisiológicos medidos no desmane e o escore de comportamento materno não permite recomendar seu uso para estimar a reatividade pósparto e a habilidade materna das ovelhas.

Palavras-chave: cordeiros, desmame, ovinos, parição, temperamento

\section{Relation between maternal behavior score with physiological characteristics of ewes}

\begin{abstract}
The objective of this study to assess the relationship between ewe maternal behavior score and physiological parameters related to their temperament. During lambing, 258 Corriedale and 50 Polwarth ewes were evaluated. They were raised on native pasture in the southwest region of Rio Grande do Sul State, Brazil. The effects of farm, age, type of lambing, maternal behavior scores and body condition score before lambing and during the weaning were evaluated in relation to heart rate, respiratory rate and body temperature of animals at weaning. In the first 24 hours after lambing, the maternal behavior score was assigned to the ewe as the distance from its lamb: 1) $\geq 10$ meters and does not return to the lamb; 2) $\geq 10$ meters from the lamb, but returns; 3) five to 10 meters; 4) between one and five meters; 5) less than one meter; 6) maintains physical contact. Ewes with maternal behavior score of $<3$ had a higher heart rate, but similar respiratory rates and body temperatures, compared with those with maternal behavior score $\geq 3$. Sheep raised on the farm in Bagé municipality had higher heart and respiratory rates. Ewes with a body condition score before lambing of $<2.0$ had lower levels of heart and respiratory rates and body temperatures. On the other hand, ewes with body condition score at weaning of $<2.0$ had the highest values of heart and respiratory rates, and the lowest body temperatures. The lack of association between the physiological parameters measured at weaning and maternal behavior does not allow for the recommendation of its use to estimate postpartum reactivity and maternal ability of ewes.
\end{abstract}

Key Words: lambing, lambs, sheep, temperament, weaning 


\section{Introdução}

O estudo do comportamento animal permite classificá-lo de acordo com seu temperamento ou reatividade (Roll et al., 2006). Animais muito reativos podem se mostrar excessivamente agitados ou nervosos na presença humana (Grandin \& Deesing, 1998) e apresentar menor ganho de peso (SilveiraBarbosa et al., 2008), baixo consumo de alimento (Brown et al., 2004; Petherick et al., 2002), além de aspectos negativos no seu comportamento reprodutivo (Dwyer, 2008).

A ovelha é descrita como um animal altamente estressado quando isolado do rebanho e facilmente assustado na presença de humanos ou predadores (Nowak, 1996). Ovinos com temperamento mais calmo podem estar associados a melhor na habilidade materna e sobrevivência de seus cordeiros (Rech et al., 2008).

O'Connor et al. (1985) desenvolveram um método para avaliar o comportamento materno de ovinos, denominado escore de comportamento materno, aplicado nas primeiras 24 horas de vida do cordeiro, com atribuição de cinco escores conforme a proximidade da ovelha ao seu cordeiro, à medida que este é manejado. As ovelhas com menores cuidados maternos direcionados aos seus cordeiros tendem a apresentar maiores taxas de mortalidade de cordeiros, principalmente nos seus primeiros dias de vida (Dwyer, 2008; Nowak et al., 2000). De acordo com esses autores, ovelhas com melhor escore de comportamento materno isolaram-se menos do rebanho no momento do parto, protegeram mais suas crias e aleitaram por período maior. A taxa de sobrevivência de seus cordeiros do nascimento ao desmame também foi maior e as ovelhas vocalizaram, com maior frequência, no momento da identificação de seus cordeiros (O'Connor, 1996; Lambe et al., 2001; Rech et al., 2008).

O aumento da eficiência econômica da produção ovina, através da redução da taxa de mortalidade dos cordeiros do nascimento até o desmame, poderia ser realizado com a seleção das ovelhas de acordo com o comportamento materno. Entretanto, a mensuração do escore de comportamento materno requer mais tempo e disponibilidade de mãode-obra. Outras medidas comportamentais e fisiológicas mensuráveis de maneira mais concentrada, rápida e economicamente viável, seriam interessantes, caso fossem relacionadas com a habilidade materna (Roll et al., 2006). Existe, porém, carência de trabalhos que relacionam o escore de comportamento materno com medidas fisiológicas do estresse.

Este trabalho foi realizado com o objetivo de avaliar a relação entre o escore de comportamento materno de ovelhas criadas na região sudoeste do Rio Grande do Sul e suas medidas fisiológicas de temperamento mensuradas no momento do desmame de seus cordeiros.

\section{Material e Métodos}

O experimento foi conduzido em três propriedades na região sudoeste do Rio Grande do Sul: uma localizada no município de Bagé, com latitude $31^{\circ} 21^{\prime} 9.8244^{\prime \prime S ~ e ~ l o n g i t u d e ~}$ $54^{\circ} 00^{\prime} 57.3624^{\prime \prime} \mathrm{W}$; durante o período de setembro a dezembro de 2006 e de setembro de 2007 a janeiro de 2008; a segunda em Dom Pedrito, com latitude $30^{\circ} 52^{\prime} 38.7984$ "S e longitude $54^{\circ} 51^{\prime} 20.217 " \mathrm{~W}$, durante o período de julho de 2008 a janeiro de 2009; e a terceira em Pedro Osório, com latitude de $31^{\circ} 55^{\prime} 33.0744^{\prime \prime S}$ e longitude de $52^{\circ} 56^{\prime} 45.9312 \mathrm{~W}$, durante o período de agosto de 2008 a fevereiro 2009.

Foram utilizadas nesse experimento 308 ovelhas, sendo 258 ovelhas da raça Corriedale (113 ovelhas em Dom Pedrito e 145 ovelhas em Bagé) e 50 da raça Ideal (em Pedro Osório). Em Dom Pedrito, as ovelhas foram encarneiradas de janeiro a abril e, em Bagé, de março a maio de 2006 e 2007. Em Pedro Osório, as ovelhas foram inseminadas em março de 2008 com sêmen de carneiro Poll Dorset. Aproximadamente 15 dias antes do parto, as ovelhas foram numeradas no flanco esquerdo e avaliadas quanto à idade e ao escore de condição corporal (Russel et al., 1969). Nesse mesmo período, as ovelhas foram dosificadas com vermífugo à base de closantel e vacinadas contra gangrena gasosa em Dom Pedrito. Em Bagé e Pedro Osório, foram dosificadas com vermífugo à base de febendazole a $8 \%$ e closantel a $10 \%$.

Depois de identificadas, as ovelhas foram transferidas para piquetes maternidades próximos às instalações, de 90 ha e 20 ha em campo natural, e 21,82 ha de pastagem de azevém, nas propriedades de Dom Pedrito, Pedro Osório e Bagé, respectivamente, para melhor controle da parição.

Os partos foram acompanhados durante o período de 22/6/2008 a 15/7/2008 em Dom Pedrito, de 24/8/2008 a 12/9/2008 em Pedro Osório e, em Bagé, de 2/9/2006 a 18/9/2006 e de 12/9/2007 a 6/10/2007. Nesses períodos, a temperatura ambiente mínima foi de $3,9^{\circ} \mathrm{C}$ e a máxima, de $26,1^{\circ} \mathrm{C}$ em Dom Pedrito; mínima de $2,5^{\circ} \mathrm{C}$ e a máxima de $28,9{ }^{\circ} \mathrm{C}$ em Pedro Osório. Em Bagé, no ano de 2006, a temperatura mínima foi de $9,0^{\circ} \mathrm{C}$ e a máxima, de $19,7^{\circ} \mathrm{C}$ e, no ano de 2007, a temperatura mínima foi de $0^{\circ} \mathrm{C}$ e a máxima, de $28,1^{\circ} \mathrm{C}$.

O desmame nas três propriedades foi feito de forma abrupta, em 8/1/2009 em Dom Pedrito, em 25/2/2009 em Pedro Osório e, em Bagé: em 2006, em 23/12/2006 e, nos animais nascidos no ano de 2007, em 19/2/2008. Os cordeiros apresentaram as seguintes idades ao desmame: 173 a 196 dias e 163 a 181 dias, respectivamente, nas propriedades de 
Dom Pedrito e Pedro Osório. No ano de 2006, na propriedade de Bagé, os cordeiros, ao desmame, apresentaram idade entre 95 e 111 dias e, em 2007, entre 133 a 157 dias.

O teste utilizado para determinar o temperamento das ovelhas durante a estação de parição foi o escore de comportamento materno, realizado de forma direta e focal entre as 12 e 24 horas de vida dos cordeiros. Esse teste consiste em atribuir escores numa escala de seis pontos, com o objetivo de avaliar a distância de fuga das ovelhas, adaptado de O'Connor et al. (1985) pela inclusão do sexto valor à ovelha que manteve contato físico com o(s) seu(s) cordeiro(s). Os cordeiros que nasceram pela manhã foram identificados e pesados à tarde e aqueles nascidos à tarde foram identificados e pesados na manhã seguinte.

Dois observadores se aproximavam da ovelha e de seu(s) cordeiro(s): um deles identificava e pesava o(s) cordeiro(os), enquanto o outro estimava visualmente a distância de fuga da ovelha, obedecendo aos seguintes escores durante o manejo de parição: 1 . a ovelha foge e permanece a mais de 10 metros quando o manejador se aproxima, não mostra interesse no(s) cordeiro(s), com ou sem vocalizações, nem retorna ao(s) cordeiro(s) durante o período de observação; 2 . a ovelha recua e permanece mais do que $10 \mathrm{~m}$ distante do(s) cordeiro(s), mas retorna ao(s) cordeiro(s) durante o período de observação; 3. a ovelha recua entre mais de 5 e menos de $10 \mathrm{~m}$ de seu(s) cordeiro(s) durante o período de observação; 4. a ovelha recua e permanece de 1 até 5 metros de seu(s) cordeiro(s), parada ou circulando em sua volta, durante o período de observação; 5. a ovelha permanece menos de um metro de seu(s) cordeiro(s), parada ou circulando em sua volta, durante o período de observação; e 6. a ovelha mantém o contato físico com o(s) seu(s) cordeiro(s) durante o período de observação.

O segundo teste utilizado para avaliar o temperamento das ovelhas foi a verificação dos parâmetros fisiológicos ao desmame. Nesse mesmo período, foi avaliado também o escore de condição corporal das ovelhas (Russel et al., 1969).

Em Dom Pedrito, a mensuração dos parâmetros fisiológicos foi realizada no início da manhã (9/1/2009), das $8 \mathrm{~h}$ às $11 \mathrm{~h} 30 \mathrm{~min}$, e a temperatura ambiental foi registrada a cada 30 minutos e variou de 22 a $25,8^{\circ} \mathrm{C}$. Em Pedro Osório, as medidas fisiológicas foram aferidas no final da tarde $(25 / 2 / 2009)$, das $16 \mathrm{~h} 30 \mathrm{~min}$ às $18 \mathrm{~h}$, e a temperatura ambiental oscilou de 24 a $25^{\circ} \mathrm{C}$, sendo registrada a cada 30 minutos. Nos animais nascidos no ano de 2006, em Bagé, as avaliações foram realizadas no período da manhã $(23 / 12 / 2006)$ das $8 \mathrm{~h}$ às 10 horas, com temperatura mínima de $19,3{ }^{\circ} \mathrm{C}$ e máxima de $21,5^{\circ} \mathrm{C}$ e, nos animais nascidos no ano de 2007 , em $19 / 02 / 2008$, das $8 \mathrm{~h}$ às $12 \mathrm{~h}$, com temperatura mínima de $17,8^{\circ} \mathrm{C}$ e máxima de $24^{\circ} \mathrm{C}$.
Foram registradas ainda a frequência cardíaca, a frequência respiratória e a temperatura corporal dos animais (Roll et al., 2006). A frequência cardíaca foi obtida com auxílio de um estetoscópio flexível colocado diretamente na região torácica esquerda à altura do arco aórtico e expressa em movimentos por minuto (movimentos/minuto). A frequência respiratória foi obtida observando-se o número de movimentos do flanco direito do animal durante 15 segundos e multiplicando o valor observado por quatro. A temperatura corporal foi determinada mediante a introdução de um termômetro clínico veterinário, com escala até $44^{\circ} \mathrm{C}$, diretamente na ampola retal do animal por um minuto.

Outros dois testes foram utilizados para avaliar o temperamento das ovelhas no momento do desmame: teste de arena e distância de fuga. No teste de arena, adaptado de Martin \& Bateson (1986), foi avaliado o grau de agitação do animal (movimentação de acordo com o número de quadrados percorridos), número de vocalizações e período de latência para iniciar o movimento, durante 30 segundos no isolamento e na presença do observador, medido utilizando-se um cronômetro.

O teste de distância de aproximação ou teste com presença humana, originalmente chamado teste de distância de fuga (adaptado de Boivin et al.,1992), foi realizado no dia do desmame, em um curral de observação. As paredes laterais do currral de observação foram cobertas com lona de polietileno, com o objetivo de isolar visualmente o animal de seus companheiros de rebanho. O piso do curral foi demarcado com barbante, em espaçamentos de $1 \mathrm{~m}^{2}$. Cada animal foi colocado no interior do curral e mantido sozinho por 30 segundos. Posteriormente, o observador entrou no curral e manteve-se imóvel junto à porteira por mais 30 segundos. A seguir, aproximou-se vagarosamente do animal, até ocorrer o primeiro deslocamento do mesmo. Registrou-se a distância (número de quadrados era igual a metros) entre o observador e o animal.

A análise estatística foi realizada considerando o delineamento inteiramente casualizado, nos qual os animais foram as unidades experimentais. Os dados de escore de condição corporal antes do parto e ao desmame e a idade das ovelhas foram categorizados para permitir seu uso como variáveis classificatórias. As ovelhas com idade igual ou inferior a quatro dentes e superior a quatro dentes $(>2$ anos), foram consideradas, respectivamente, novas e maduras. Os escores de condição corporal das ovelhas antes do parto e ao desmame foram classificados como baixo, intermediário e bom quando os valores foram: iguais ou inferiores a 1,5, entre 2,0 e 2,5 e maiores que 2,5 , respectivamente.

As ovelhas que apresentaram escore de comportamento materno inferior a 3 (escore de comportamento materno 
ruim) foram consideradas as piores mães e aquelas com escore de comportamento materno igual ou superior a três foram consideradas boas mães (escore de comportamento materno bom).

Os valores de temperatura corporal, frequência cardíaca e frequência respiratória não apresentaram distribuição normal, mesmo quando transformados pela aplicação da raiz quadrada e, posteriormente, pela aplicação logarítmica. Devido a esse fato, realizou-se uma análise não-paramétrica para essas variáveis, pelo procedimento NPAR1WAY do SAS (Statistical Analysis System, versão 8.1), utilizando-se o teste de Wilcoxon/Kruskal-Wallis, com o objetivo de avaliar o efeito do escore de comportamento materno $(n=2)$, propriedade $(n=3)$, escore de condição corporal das ovelhas antes do parto $(\mathrm{n}=2)$ e escore de condição corporal das ovelhas ao desmame $(\mathrm{n}=2)$, tipo de parto $(\mathrm{n}=2)$ e idade da ovelha ( $\mathrm{n}=2)$, conforme o modelo matemático representado abaixo:

Yijlmnop $=\mu+\mathrm{Ti}+\mathrm{Ij}+\mathrm{ECPk}+\mathrm{ECCDl}+\mathrm{TPm}+\mathrm{Pn}+$ eijklmno, em que: Yijklmnop=1-ésima observação, no i-ésimo escore de comportamento materno, na j-ésima idade, no k-ésimo escore de condição corporal antes do parto, no l-ésimo escore de condição corporal ao desmame, no m-ésimo tipo de parto e na n-ésima propriedade; $\mu=$ média geral do experimento; $\mathrm{Ti}=$ efeito do i-ésimo escore de comportamento materno $(\mathrm{GL}=1) ; \mathrm{Ij}=$ efeito do $\mathrm{j}$-ésima idade $(\mathrm{GL}=1) ; \mathrm{ECPk}=$ efeito do k-ésimo escore de condição corporal antes do parto $(\mathrm{GL}=1) ; \mathrm{ECCDl}=$ efeito do l-ésimo escore de condição corporal ao desmame $(\mathrm{GL}=1) ; \mathrm{TPm}=$ efeito do $\mathrm{m}$-ésimo tipo de parto $(\mathrm{GL}=1) ; \mathrm{Pn}=$ efeito da $n$-ésima propriedade; eijklmno= n-ésimo erro, associado à ijklmnop-ésima observação.

Complementarmente, foram utilizados os procedimentos PROC UNIVARIATE, CORR (coeficientes de Spearman) e REG para, respectivamente, realizar análise descritiva/teste de normalidade e verificar a existência de relações entre as variáveis fisiológicas e o escore de comportamento materno. Adotou-se a probabilidade de 0,05 para rejeição da hipótese de nulidade.

\section{Resultados e Discussão}

Observou-se maior proporção de ovelhas com parto simples, escore de comportamento materno $<3$, escores de condição corporal da ovelha antes do parto e ao desmame entre 1,0 e 1,5 e maior número de ovelhas com idade superior a 2 anos (Tabela 1).

O menor número de ovelhas com partos gemelares deve-se, possivelmente, a um escore de condição corporal baixo na época de cobertura. Observou-se também maior número de ovelhas com escores de condição corporal antes do parto e ao desmame entre 1,0 e 1,5, principalmente no momento do desmame, o que pode ser explicado pelo fato de as ovelhas terem sido criadas extensivamente em campo natural com pouca disponibilidade de forragem durante a gestação e no período de aleitamento.

Embora tenha sido observada porcentagem maior de escore de comportamento materno inferior a 3 e de ovelhas com idade superior a 2 anos, esses resultados são reflexos do tipo de criação extensiva e da falta de experiência prévia, já que foi a primeira vez que houve intervenção humana no manejo de parição nessas propriedades, e/ou pelo pouco contato do homem com as ovelhas, caracterizando intensa reação de medo. Segundo Nowak (1996), ovelhas mais reativas permanecem menos tempo no local do parto e demonstram pior comportamento materno, o que foi constatado nesse experimento.

Ovelhas com escore de comportamento materno inferior a 3 apresentaram maior frequência cardíaca $(P=0,049)$ se comparadas àquelas com escore de comportamento materno igual ou superior a 3. Entretanto, a frequência respiratória ( $\mathrm{P}=0,857)$ e a temperatura corporal $(\mathrm{P}=0,805)$ das ovelhas não variaram conforme o seu escore de comportamento materno.

A idade não influenciou os valores de frequência cardíaca ( $\mathrm{P}=0,730)$, frequência respiratória $(\mathrm{P}=0,430)$ e temperatura corporal $(P=0,904)$, embora animais mais jovens apresentem maior taxa metabólica. O tipo de parto interferiu apenas na frequência respiratória. Ovelhas com parto gemelar tiveram maior frequência respiratória em comparação àquelas com parto simples, 70,61 vs. 64,65 movimentos/minuto (Tabela 2). $\mathrm{O}$ aumento na frequência respiratória é consequência da restrição na expansão pulmonar, devido à compressão do útero, principalmente em partos múltiplos, sobre o diafragma, o qual se apresenta limitado durante a inspiração. Esse aumento tem como finalidade garantir ventilação adequada ao animal (Swenson \& Reece, 1996).

Ovelhas com baixo escore de condição corporal antes do parto apresentaram menores médias de frequência cardíaca, frequência respiratória e temperatura corporal $(\mathrm{P}<0,05)$ em comparação àquelas com escore de condição corporal intermediário ou bom. Por outro lado, ovelhas com baixo escore de condição corporal ao desmame evidenciaram valores maiores de frequência cardíaca e frequência respiratória, mas menor valor de temperatura corporal $(\mathrm{P}<0,05)$ (Tabela 2).

A relação entre os valores de escore de condição corporal antes do parto e os maiores valores dos parâmetros fisiológicos, $>1,5$ e a relação inversa observada entre os valores dos parâmetros fisiológicos e escore de condição corporal medida ao desmame (Tabela 2) podem ser 
parcialmente explicadas pela relação negativa entre o escore de condição corporal antes do parto e ao desmame $(\mathrm{r}=-0,16 ; \mathrm{P}=0,0382 ; \mathrm{n}=175)$.

As ovelhas com maior escore de condição corporal antes do parto foram mais reativas no teste de arena: demonstraram maior movimentação (correlação positiva com a distância percorrida no teste de arena: $\mathrm{r}=0,33 ; \mathrm{P}=0,0001$; vocalizaram mais no isolamento, $\mathrm{r}=0,34 ; \mathrm{P}=0,0001$; apresentaram menor latência para iniciar a movimentação na presença do observador: $\mathrm{r}=-0,23 ; \mathrm{P}=0,0029$; movimentaram-se mais na presença do observador (correlação positiva com a distância percorrida na presença do observador: $r=0,39$;
$\mathrm{P}=0,0001)$ e apresentaram maior distância de fuga: $\mathrm{r}=0,37$; $\mathrm{P}=0,0001$.

Essa maior movimentação se refletiu em maiores valores de frequência cardíaca e frequência respiratória, confirmando a maior reatividade das ovelhas com maior escore de condição corporal.

Por ouro lado, as ovelhas com maior escore de condição corporal ao desmame apresentaram menor movimentação durante o teste de arena na fase de isolamento social (correlação negativa com a distância percorrida: $r=-0,26$; $\mathrm{P}=0,0006$; correlação positiva com o tempo de latência para iniciar o movimento: $\mathrm{r}=0,16 ; \mathrm{P}=0,0332$ ) e durante a fase em

Tabela 1 - Frequências relativas (\%) das ovelhas dentro de cada classe

\begin{tabular}{|c|c|c|c|c|c|c|c|c|}
\hline Classe & Parto & Classe & $\mathrm{ECM}^{1}$ & Classe & $\mathrm{ECCP}^{2}$ & $\mathrm{ECCD}^{3}$ & Classe & Idade \\
\hline Simples & 75,97 & $<3$ & 73,03 & $1,0-1,5$ & 46,10 & 72,73 & $\leq 2$ & 12,01 \\
\hline Gemelar & 24,03 & $\geq 3$ & 26,95 & $\begin{array}{c}2,0-2,5 \\
>2,5\end{array}$ & $\begin{array}{l}33,77 \\
20,13\end{array}$ & $\begin{array}{c}18,83 \\
8,44\end{array}$ & $>2$ & 87,99 \\
\hline
\end{tabular}

${ }^{1}$ ECM - escore de comportamento materno.

${ }^{2}$ ECCP - escore de condição corporal da ovelha antes do parto.

${ }^{3}$ ECCD - escore de condição corporal da ovelha ao desmame.

Tabela 2 - Valores médios dos parâmetros fisiológicos em relação às variáveis estudadas

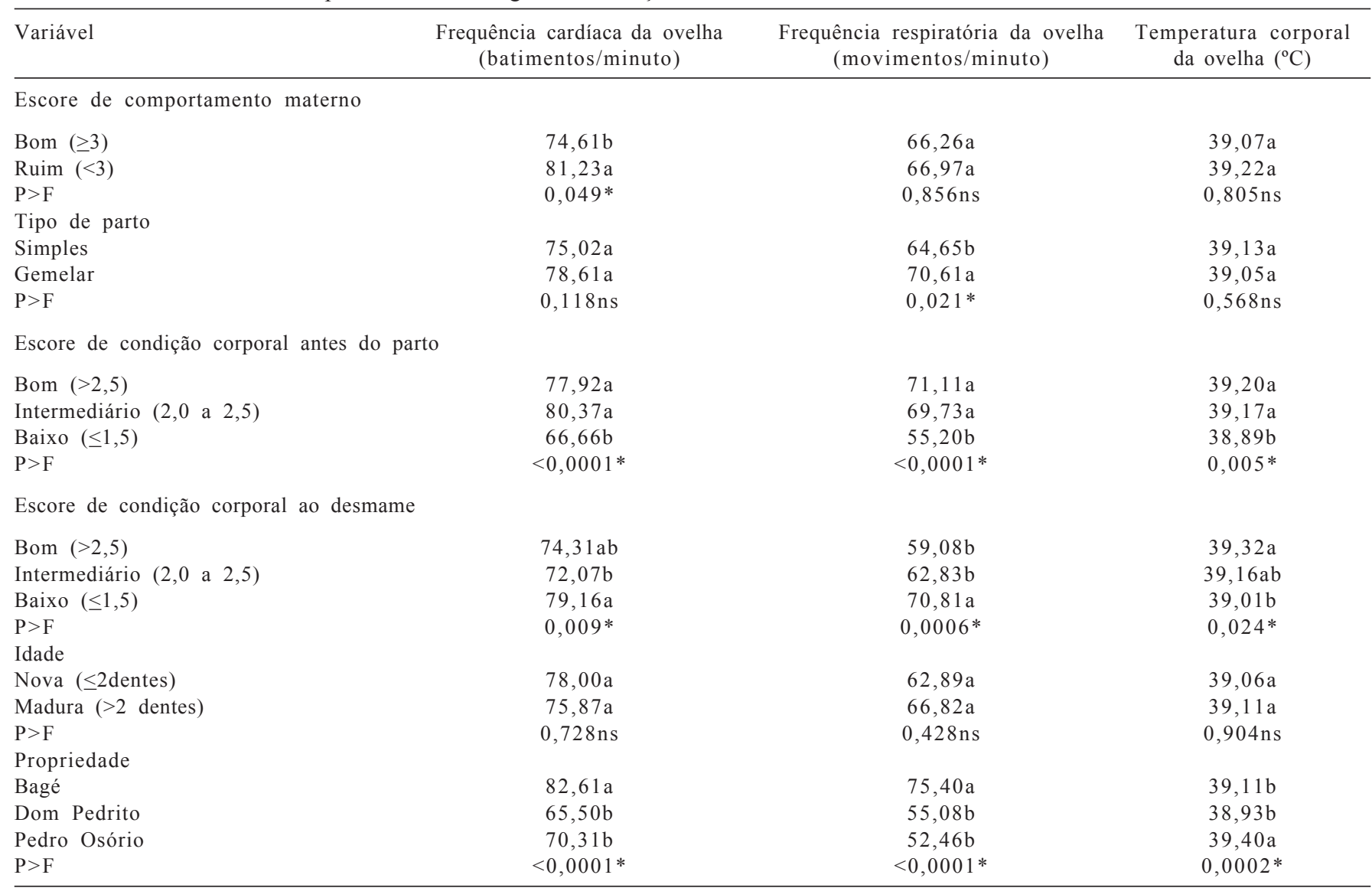

*Valores com $\mathrm{P}<0,05$ foram considerados significativos no teste de Wilcoxon.

ns Valores com $\mathrm{P}>0,05$ não foram significativos no teste de Wilcoxon.

a e b: médias na mesma coluna seguidas por letras distintas são diferentes $(P<0,05)$ pelo teste de DMS Fisher. 
presença do observador (correlação negativa com a distância percorrida na presença do observador: $r=-0,21$; $\mathrm{P}=0,0064$; correlação positiva com latência para iniciar o movimento: $\mathrm{r}=0,20 ; \mathrm{P}=0,0124$; e mantiveram menor distância do observador quando esse tentou se aproximar (correlação negativa com distância de fuga: $\mathrm{r}=-0,29 ; \mathrm{P}=0,003)$. Nesse segundo caso, as ovelhas com maior escore de condição corporal ao desmame reagiram movimentando-se menos no teste de arena, o que provocou menor valor das variáveis fisiológicas.

Entretanto, a relação entre escore de condição corporal e o comportamento no teste de arena não está clara. De acordo com alguns autores (Kilgour, 1998; Kilgour \& Szantar-Coddington, 1995), animais mais pesados teriam maior dificuldade de se movimentar nos testes. Como o teste de arena foi aplicado durante o desmame, esse fato pode ter ocorrido com os animais com maior escore de condição corporal ao desmame, enquanto aqueles com maior escore de condição corporal antes do parto podem ter modificado seu escore de condição corporal pelo intervalo de tempo decorrido entre essa medida e as avaliações do comportamento.

No entanto, os valores de frequência cardíaca $(\mathrm{P}<0,0001)$, frequência respiratória $(\mathrm{P}<0,0001)$ e temperatura corporal $(P=0,0003)$ variaram conforme a propriedade. Nas ovelhas criadas em Bagé, foram maiores os valores de frequência cardíaca e frequência respiratória, enquanto nas ovelhas criadas na propriedade de Pedro Osório foi observado o maior valor de temperatura corporal. Os maiores valores observados em Bagé em relação às demais propriedades permitem associar o aumento desses parâmetros fisiológicos, normalmente associados à presença de vocalizações, à maior agitação, ao medo, à angústia e à emoção intensa dos animais nessa propriedade (Romeyer \& Bouissou, 1992; Murphy et al., 1998; Boissy et al., 2005; Reefmann et al., 2009).

Essas diferenças podem ser explicadas pelo manejo mais aversivo habitualmente realizado pelos funcionários na propriedade localizada em Bagé, acarretando em experiência prévia negativa e fomentando aversão dos animais à presença humana. Nessa propriedade, os animais eram conduzidos no campo de forma rápida, com vocalizações humanas abruptas e de alta intensidade (gritos), uso de cães destreinados e muito agitados em comparação às outras duas propriedades estudadas.

A intensidade e a duração do estímulo estressante determinam a extensão do tempo requerido para que os parâmetros fisiológicos retornem ao nível normal, sendo necessários mais de 30 minutos em situações de estresse severo (Stermer et al., 1981).

Esse aumento poderia, também, estar relacionado à temperatura ambiente elevada (Silva \& Gondim, 1971).
Entretanto, ao avaliar o tipo de propriedade, a temperatura ambiente não interferiu nos resultados desse experimento, sendo semelhantes nas fazendas avaliadas. Além disso, os menores valores numéricos da temperatura ambiental foram registrados na propriedade de Bagé durante a realização as medições em relação às demais propriedades. Ressalta-se que todas as medidas fisiológicas foram realizadas dentro da faixa de conforto térmico de ovinos e que o manejo dos animais durante a realização das medidas foi feito de forma tranquila, reduzindo o estresse dos animais.

Ovelhas que pariram mais de um cordeiro tiveram frequência respiratória mais elevada, o que poderia ser explicado por uma maior taxa metabólica desses animais em relação a ovelhas com parto simples. Todavia, não foi observada diferença significativa entre os valores de frequência cardíaca.

Segundo Kolb (1980), a frequência cardíaca dos ovinos é de 70 a 80 batimentos/minuto, a temperatura corporal deve estar entre $39 \mathrm{e} 41^{\circ} \mathrm{C}$ e a frequência respiratória entre $16 \mathrm{e}$ 34 movimentos/minuto, como reportou Reece (1996). Neste estudo, observou-se aumento considerável, acima dos parâmetros fisiológicos normais, na frequência respiratória dos animais, observado por meio de uma respiração mais curta e superficial, sem aumento considerável na frequência cardíaca. Isso, possivelmente, em consequência de uma situação potencialmente estressante para a ovelha, decorrente de sua separação dos seus companheiros de rebanho e sua contenção para a realização da medida.

Os animais mais suscetíveis ao estresse podem apresentar aumento dos valores dessas características, mesmo quando sua movimentação for restrita e a temperatura ambiente for amena (Lynch et al., 1992; Hutson, 2000; Roussel et al., 2004; Reefmann et al., 2009). Portanto, quanto maior a atividade física do animal e quanto mais ele for confrontado a situações de medo, maiores serão suas frequências cardíaca e respiratória, com o propósito de aumentar a concentração de sangue (nutrientes e oxigênio) no músculo e no cérebro, permitindo, também, ao animal decidir entre fugir ou lutar, devido à reação de defesa ou alarme (Cunningham \& Klein, 2009). Isso justificaria, parcialmente, o aumento no valor de frequência respiratória encontrado nesse experimento, acima dos parâmetros fisiológicos normais nessa espécie. Em outros estudos, foi constatado aumento na frequência cardíaca dos ovinos em decorrência do isolamento visual, transporte, introdução a um novo rebanho, aproximação do ser humano e à aproximação deste acompanhado de um cão (Baldock \& Sibly, 1990) e durante a tosquia (Webster \& Lynch, 1966).

O aumento da frequência cardíaca encontrado nos animais com escore de comportamento materno inferior a 
três está relacionado ao fato de elas manifestarem maior aversão à presença humana no momento da identificação dos cordeiros e permanecerem mais afastadas destes. Também, demonstraram maior aversão ao contato com o homem, comprovada pelo aumento da sua frequência cardíaca, quando as ovelhas foram separadas de seus cordeiros e de suas companheiras de rebanho e quando foram imobilizadas para a realização das medições dos parâmetros fisiológicos.

Todavia, não se tem explicação para a ausência de efeito do escore de comportamento materno sobre frequência respiratória e temperatura corporal, visto que normalmente essas medidas são positivamente correlacionadas, já que os testes aplicados apresentam-se associados às reações de medo, angústia, emoção intensa e agitação nos animais (Romeyer \& Bouissou, 1992; Murphy et al., 1998; Boissy et al., 2005; Reefmann et al., 2009). No presente estudo, os parâmetros fisiológicos foram positiva e moderadamente correlacionados: frequência cardíaca $\times$ temperatura corporal $(\mathrm{r}=0,35 ; \mathrm{P}<0,0001)$, frequência cardíaca $\times$ frequência respiratória $(\mathrm{r}=0,56 ; \mathrm{P}<0,0001)$, apesar de a temperatura corporal $\times$ frequência respiratória $(\mathrm{r}=0,17 ; \mathrm{P}=0,0225)$ apresentarem correlação positiva e fraca.

A ausência de efeito da idade sobre os parâmetros fisiológicos nesse experimento permitiu constatar a independência da idade dos animais em relação aos parâmetros fisiológicos avaliados. Embora tenham sido observados valores de frequência cardíaca sem diferenças significativas, foi constatado um número superior de batimentos cardíacos nos animais mais jovens (78 vs. 75 batimentos cardíacos/minuto). Grandin (1993) observou que animais mais jovens apresentaram maiores valores dos parâmetros fisiológicos, indicando maior suscetibilidade aos agentes estressantes, o que foi creditado à falta de experiência prévia e à maior taxa metabólica (Reece, 2005). Montano et al. (2008) e Rech et al. (2008), trabalhando, respectivamente, com ovelhas da raça Crioula e Corriedale $\times$ Ideal, observaram maiores valores de frequência cardíaca, frequência respiratória e temperatura corporal em animais mais jovens. Ovinos jovens movimentam-se mais em ambientes novos (pista de remate) ovinos mais velhos, fato atribuído à sua menor experiência prévia e maior resistência à condução pelo homem (Barbosa Silveira et al., 2010).

Embora tenham sido detectadas diferenças entre os valores dos parâmetros fisiológicos, os valores de temperatura corporal e frequência cardíaca mantiveram-se dentro dos padrões normais (Cunningham \& Klein, 2009). Os valores de frequência respiratória foram superiores aos reportados como normais, dentro da faixa de conforto para os ovinos.

Não foram detectadas correlações lineares significativas do escore de comportamento materno e da idade das ovelhas em relação aos valores de temperatura corporal, frequência cardíaca e frequência respiratória, mas foram detectadas relações lineares e positivas entre essas variáveis e valores de escore de condição corporal antes do parto, mas negativas com os valores de escore de condição corporal ao desmame (Tabela 3), o que confirma os resultados obtidos na análise de variância.

A análise de regressão linear múltipla do escore de comportamento materno em relação às variáveis fisiológicas comprovou que o escore de comportamento materno foi influenciado, de forma negativa, pela frequência cardíaca e pelo escore de condição corporal ao desmame, porém o $\mathrm{r}^{2}$ do modelo foi muito baixo $\left(\mathrm{r}^{2}=0,04\right)$, explicando somente $4 \%$ da variação (escore de comportamento materno $=$ 4,57 - 0,01 frequência cardíaca $-0,27$ escore de condição corporal ao desmame, $\mathrm{P}=0,0237$ ). O comportamento materno foi negativamente influenciado pelo temperamento mais reativo das ovelhas, nesse caso manifestado pela frequência cardíaca e também pela condição nutricional das ovelhas, na qual aquelas com menor escore de condição corporal ao desmame foram mais reativas.

Essas relações negativas se referem, em parte, ao fato de que ovelhas mais reativas tendem a manifestar reação de medo e de aversão à presença humana mais intensa, o que influencia o escore de comportamento materno. Escores extremos de condição corporal (ovelhas obesas e magras) foram associados a menores escores de comportamento materno por outros autores e a modificações comportamentais, como menos tempo gasto em atividades como lamber e

Tabela 3 - Coeficientes de correlação linear entre os parâmetros fisiológicos medidos ao desmame com a idade, o escore de comportamento materno e os escores de condição corporal da ovelha

\begin{tabular}{|c|c|c|c|}
\hline Variável & $\begin{array}{l}\text { Frequência cardíaca da ovelha } \\
\text { (batimentos/minuto) }\end{array}$ & $\begin{array}{l}\text { Frequência respiratória da ovelha } \\
\text { (movimentos/minuto) }\end{array}$ & $\begin{array}{c}\text { Temperatura corporal } \\
\text { da ovelha }\left({ }^{\circ} \mathrm{C}\right)\end{array}$ \\
\hline Escore de comportamento materno & $-0,032 \mathrm{~ns}$ & $0,083 \mathrm{~ns}$ & $-0,061 \mathrm{~ns}$ \\
\hline Escore de condição corporal antes do parto & $0,295 *$ & $0,370 *$ & $0,182 *$ \\
\hline Escore de condição corporal ao desmame & $-0,243 *$ & $-0,270 *$ & $0,140 \mathrm{~ns}$ \\
\hline Idade & $0,125 \mathrm{~ns}$ & $0,091 \mathrm{~ns}$ & $-0,090 \mathrm{~ns}$ \\
\hline
\end{tabular}

* Valores com $\mathrm{P}<0,05$ foram considerados significativos no teste de Spearman.

ns Valores com $\mathrm{P}>0,05$ não foram significativos no teste no teste de Spearman. 
cuidar do cordeiro e emitir menor número de vocalizações (Dwyer et al., 2003; Mariz et al., 2007).

Entretanto, além do relativo pequeno número de animais, existe grande variação na manifestação desse comportamento, em parte modulado por fatores, como experiência prévia, reatividade ou temperamento, diferenças raciais no cuidado com os cordeiros e na reatividade à presença humana, condições ambientais, como clima, estado de condição corporal da ovelha e o próprio comportamento do cordeiro, que explicam o baixo coeficiente de determinação da regressão.

Ao comparar os parâmetros fisiológicos mensurados nos cordeiros e nas ovelhas, observou-se correlação positiva significativa entre a frequência cardíaca $(r=0,39)$ e a frequência respiratória $(\mathrm{r}=0,32)$ das ovelhas e dos cordeiros, apesar de os valores de temperatura corporal das ovelhas e dos seus cordeiros não terem sido correlacionados (Tabela 4). Os cordeiros filhos de ovelhas com maiores frequência cardíaca e frequência respiratória apresentaram maiores frequência cardíaca e frequência respiratória ao desmame, indicando que ovelhas mais reativas geraram cordeiros mais reativos, o que pode ser creditado à influência genética da ovelha progenitora, e não à influência do genótipo das ovelhas que apenas criaram cordeiros filhos de outras ovelhas (Bickell et al., 2009). A herdabilidade do comportamento materno variou de baixa a moderada, ou seja, de 0,09 a 0,13 (Lambe et al., 2001); 0,09 a 0,32 (Everett-Hincks et al., 2005); e 0,25 a 0,50 (Bussab, 1998).

Tabela 4 - Coeficientes de correlação linear entre os parâmetros fisiológicos das ovelhas e dos cordeiros ao desmame

\begin{tabular}{|c|c|c|c|}
\hline Variável & $\begin{array}{l}\text { Frequência cardíaca da ovelha } \\
\text { (batimentos/minuto) }\end{array}$ & $\begin{array}{l}\text { Frequência respiratória da ovelha } \\
\text { (movimentos/minuto) }\end{array}$ & $\begin{array}{c}\text { Temperatura corporal } \\
\text { da ovelha }\left({ }^{\circ} \mathrm{C}\right)\end{array}$ \\
\hline $\begin{array}{l}\text { Frequência cardíaca do cordeiro } \\
\text { (batimentos/minuto) }\end{array}$ & $0,390 \mathrm{~ns}$ & $0,421 \mathrm{~ns}$ & $0,20 \mathrm{~ns}$ \\
\hline $\begin{array}{l}\text { Frequência respiratória do cordeiro } \\
\text { (movimentos/minuto) }\end{array}$ & $0,155^{*}$ & $0,32 *$ & $0,06^{*}$ \\
\hline Temperatura corporal do cordeiro $\left({ }^{\circ} \mathrm{C}\right)$ & $-0,120 *$ & $-0,02 *$ & $0,070 \mathrm{~ns}$ \\
\hline
\end{tabular}

* Valores com $\mathrm{P}<0,05$ foram considerados significativos no teste de Spearman.

ns Valores com $\mathrm{P}>0,05$ não foram significativos no teste no teste de Spearman.

\section{Conclusões}

O temperamento das ovelhas medido por meio de seus parâmetros fisiológicos no momento do desmame dos cordeiros não se correlaciona de forma global com o escore de comportamento materno, o que não permite recomendar a substituição do teste de escore de comportamento materno pelos parâmetros fisiológicos (temperatura corporal, frequência cardíaca e frequência respiratória) avaliados antes do parto e ao desmame em ovelhas.

\section{Referências}

BALDOCK, N.M.; SIBLY, R.M. Effects of handling and transportation on the heart rate and behaviour of sheep. Applied Animal Behaviour Science, v.28, n.1-2, p.15-39, 1990.

BARBOSA SILVEIRA, I.; FISCHER, V.; MENDONÇA, G. Efeito do genótipo e da idade de ovinos na reatividade medida em pista de venda. Revista Brasileira de Zootecnia, v.39, n.10, p.2304-2309, 2010.

BICKELL, S.L.; POINDRON, P.; NOWAK, R. et al. Genotype rather than non-genetic behavioural transmission determines the temperament of Merino lambs. Animal Welfare, v.18, p.459-466, 2009.

BOISSY, A.; BOUIX, J.; ORGEUR, P. et al. Genetic analysis of emotional reactivity in sheep: effects of the genotypes of the lambs and of their dams. Genetics Selection Evolution, v.37, n.5, p.381-401, 2005.

BUSSAB, V.S.R. Uma abordagem psicoetológica do comportamento materno. In: PARANHOS DA COSTA, M.J.R.; CROMBERG, V.U. (Eds.) Comportamento materno em mamíferos: bases teóricas e aplicações aos ruminantes domésticos. [S.I.]: SBEt, 1998. p.17-30.

BROWN, E.G.; CARSTENS, G.E.; FOX, J.T. et al. [2004]. Relationships between temperament and performance traits of growing calves. In: Beef Cattle Research in Texas Publication, section Physiology. Disponível em: <http:// www.animalscience.tamv.edu/ansc/beef/bcrt/2004/brown $>$. Acesso em: 14 jan. 2008.

CUNNINGHAM, J.G.; KLEIN, B.G. Fisiología veterinaria Barcelona: Elsevier, 2009. 700p.

DWYER, C.M.; LAWRENCE, A.B.; BISHOP, S.C. et al. Ewe-lamb bonding behaviours at birth are affected by maternal undernutrition in pregnancy. British Journal of Nutrition, v.89, n.1, p.123-136, 2003.

DWYER, C.M. Genetic and physiological determinants of maternal behavior and lamb survival: Implications for low-input sheep management. Journal of Animal Science, v.86, n.14, p.E259E270, 2008.

EVERETT-HINCKS, J.M.; LOPEZ-VILLALOBOS, N.; BLAIR, H.T et al. The effect of maternal behavior score on Lamb and litter survival. Livestock Production Science, v.93, n.1, p.51-61, 2005.

GRANDIN, T. Behavioral agitation during handling of cattle is persistent over time. Applied Animal Behaviour Science, v.36, n.1, p.1-9, 1993.

GRANDIN, T.; DEESING, M.J. La genética del Comportamiento Animal. In: GRANDIN, T. (Ed.). Genetics and the behavior of domestic animals. San Diego: Academic Press, 1998 
Disponível em: <http://www.grandin.com/spanish//genetica. comportamiento.html>. Acesso em: 28 jan. 2010.

HUTSON, G.D. Behavioural principles of sheep handling. In: GRANDIN, T. (Ed.). Livestock handling and transport. Wallingford: CAB International, 2000. p.175-200.

KOLB, E. Fisiologia veterinária. Rio de Janeiro: Guanabara, 1980. 612p.

KILGOUR, R.J. Arena behaviour is a possible selection criterion for lamb-rearing ability; it can be measured in young rams and ewes. Applied Animal Behaviour Science, v.57, p.81-89, 1998.

KILGOUR, R.J.; SZANTAR-CODDINGTON, M.R. Arena behavior of ewes selected for superior mothering ability differs from that of unselected ewes. Animal Reproduction Science, v.37, p.133-141, 1995.

LAMBE, N.R.; CONINGTON, J.; BISHOP, S.C. et al. A genetic analysis of maternal behaviour score in Scottish Blackface sheep. Animal Science, v.72, n.2, p.415-425, 2001.

LYNCH, J.J.; HINCH, G.N.; ADAMS, D.B. The behaviour of sheep: biological principles and implications for production. Wallingford, Oxon: CAB International, 1992. 237p.

MARIZ, T.M.A.; PIMENTA FILHO, E.C.; MEDEIROS, A.N. et al. Relação materno-filial da raça Morada Nova recebendo dietas com três níveis de energia, ao final da gestação. Revista Brasileira de Zootecnia, v.36, n.6, p.1889-1893, 2007.

MONTANO, D.B.; FISCHER, V.; PEGORARO, E.J. et al. Comportamento materno-filial de dois ecotipos da raça crioula e sua cruza mantidos em sistema semiextensivo no Município de Eldorado do Sul - RS. In: ENCONTRO ANUAL DE Etologia, 26., 2008, Poços de Caldas. Anais... Poços de Caldas: Sociedade Brasileira de Etologia, [2008]. (CD-ROM).

MURPHY, P.M.; LINDSAY, D.R.; LE NEINDRE, P. Temperament of Merino ewes influences maternal behaviour and survival of lambs. In: CONGRESS OF THE INTERNATIONAL SOCIETY FOR APPLIED ETHOLOGY, 32., 1998, Clermont-Ferrand, France. Proceedings... Clermont-Ferrand: INRA, 1998. p.131.

NOWAK, R. Neonatal survival: contributions from behavioural studies in sheep. Applied Animal Behaviour Science, v.49, n.1, p.61-72, 1996.

NOWAK, R.; PORTER, R.H.; LÉVY, F. et al. Role of mother-young interactions in the survival of offspring in domestic mammals. Reviews of Reproduction, v.5, n.3, p.153-163, 2000.

O'CONNOR, C.E. Ewe maternal behaviour score and lamb growth: Ten years on. Proceedings of the New Zealand Society of Animal Production, v.56, p.107-109, 1996.

O'CONNOR, C.E.; JAY, N.P.; NICOL, A.M. et al. Ewe maternal behaviour score and lamb survival. Proceedings of the New Zealand Society of Animal Production, v.45, p.159-162, 1985 .
PETHERICK, J.C.; HOLROYD, R.G.; DOOGAN, V.J. Productivity, carcass and meat quality of a lot fed Bos indicus cross steers grouped according to temperament. Australian Journal of Experimental Agriculture, v.42, n.4, p.389-398, 2002.

RECH, C.L.S.; RECH, J.L.; FISCHER, V. et al. Temperamento e comportamento materno-filial de ovinos das raças Corriedale e Ideal e sua relação com a sobrevivência dos cordeiros. Ciência Rural, v.38, n.5, p.1388-1393, 2008.

REECE, W.O. Fisiologia de animais domésticos. São Paulo: Roca, 1996. p.137-254.

REECE, W.O. Functional anatomy and physiology of domestic animals. Baltimore: Lippincott Williams \& Wilkins, 2005. $513 \mathrm{p}$.

REEFMANN, N.; KASZAS, F.B.; WECHSLER, B. et al. Physiological expression of emotional reactions in sheep. Physiology \& Behavior, v.98, p.235-241, 2009.

ROLL, V.F.B.; RECH, C.L.S.; XAVIER, E.G. et al. Comportamento animal: conceitos e técnicas de estudo. Pelotas: Editora e Gráfica da UFPel, 2006. 109p.

ROMEYER, A.; BOUISSOU, M.F. Assessment of fear reactions in domestic sheep, and influence of breed and rearing conditions. Applied Animal Behaviour Science, v.34, n.1, p.93-119, 1992.

ROUSSEL S.; HEMSWORTH P.H.; BOISSY, A. et al. Effects of repeated stress during pregnancy in ewes on the behavioural and physiological responses to stressful events and birth weight of their offspring. Applied Animal Behaviour Science, v.85, n.3-4, p.259-276, 2004.

RUSSEL, A.J.F.; DONEY, J.M.; GUNN, R.G. Subjective assessment of body fat in live sheep. The Journal Agricultural Science, v.72, n.3, p.451-454, 1969.

SILVA, R.G.; GONDIM, A.G. Comparação entre as raças Sindi e Jersey e seus mestiços, relativamente à tolerância ao calor na região Amazônica. Pesquisa Agropecuária Brasileira, v.6, p.37-44, 1971 .

SILVEIRA-BARBOSA, I.; FISCHER, V.; FARINATTI, L.H.E. et al. Relação entre genótipos e temperamento de novilhos Charolês $\mathrm{x}$ Nelore em confinamento. Revista Brasileira de Zootecnia, v.37, n.10, p.1808-1814, 2008.

STERMER, R.A.; CAMP, T.H.; STEVENS, D.G. Feeder cattle stress during handling and transportation. American Society of Agricultural Engineers, n.81-6001, p.246-249, 1981.

SWENSON, M.J.; REECE, W.O. Dukes fisiologia de animais domésticos. 11.ed. Rio de Janeiro: Guanabara Koogan, 1996. $856 \mathrm{p}$.

WEBSTER, M.E.D.; LYNCH, J.J. Some physiological and behaviour consequence of shearing. Proceedings of the Australian Society of Animal Production Bull, v.6, p.234-239, 1966. 